Check for updates

Cite this: RSC Adv., 2018, 8, 16762

\title{
Modifying collagen with alendronate sodium for bone regeneration applications $\dagger$
}

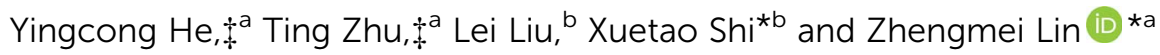

Phosphorylated materials are attractive candidates for bone regeneration because they may facilitate the construction of a phosphorylated bone extracellular matrix (ECM) to build a beneficial environment for bone formation. Here, we designed and synthesized a new phosphorylated material, collagen type I phosphorylated with alendronate sodium (Col-Aln), based on the biodegradable osteoconductive collagen backbone. Col-Aln can distinctly accelerate in vitro mineralization in simulated body fluid. ColAln showed good biocompatibility with bone marrow mesenchymal stem cells (BMSCs) and promoted their adhesion as well as the osteogenic differentiation of BMSCs more effectively than did pure collagen. Furthermore, collagen and Col-Aln scaffolds implanted into a critical-sized rat cranial defect for 4 and 8 weeks were shown to degrade in vivo and helped to facilitate bone growth in the defect, while the phosphate-containing Col-Aln scaffold significantly promoted new bone formation. Col-Aln provides a new strategy to integrate bioactive phosphate molecules via covalent grafting onto biopolymers and has promise for bone regeneration applications.
\end{abstract}

Received 3rd March 2018

Accepted 26th April 2018

DOI: $10.1039 / \mathrm{c} 8 \mathrm{ra01872c}$

rsc.li/rsc-advances

phosphorylated polymers are limited in biodegradability

\section{Introduction}

Scaffolds for bone tissue engineering play a great role in repairing bone defects resulting from trauma, tumours, and congenital diseases. ${ }^{1-4}$ This often causes there to be a crucial demand for scaffold materials, as scaffolds have been a widely investigated and applied strategy for bone regeneration. As a key component, scaffolds need to possess properties to support cells in proliferation, osteogenic differentiation, and bone formation in vivo. ${ }^{5}$ From the perspective of bionics, phosphorylated materials are expected to be the new generation of materials by mimicking the microenvironment of bone, a highly phosphorylated tissue. Recent studies have focused on phosphorylated polymers with potential biomimetic bioactivity derived from phosphate. ${ }^{6-8}$ Phosphorylated polymers are similar to the core of phosphorylated proteins and can thereby efficiently promote mineralization and mimic the microenvironment required for formation of organic/inorganic hybrid bone. ${ }^{9}$ With the process of degradation, phosphorylated compositions could release phosphate, which is crucial for ECM mineralization and ossification. ${ }^{\mathbf{1 0}}$ However, some

${ }^{a}$ Department of Operative Dentistry and Endodontics, Guanghua School of Stomatology, Sun Yat-sen University, Guangdong Provincial Key Laboratory of Stomatology, Guangzhou, 510055, China.E-mail:linzhm@mail.sysu.edu.cn

${ }^{b}$ National Engineering Research Centre for Tissue Restoration and Reconstruction, School of Material Science and Engineering, South China University of Technology, Guangzhou, 510640, PR China. E-mail: shxt@scut.edu.cn

$\dagger$ Electronic supplementary information (ESI) available. See DOI: $10.1039 / \mathrm{c} 8 \mathrm{ra} 01872 \mathrm{c}$

$\$$ These authors contribute equally to this paper. because of the structure of their carbon-carbon backbones, such as with polymethacrylate and polyvinyl alcohol moieties. ${ }^{\mathbf{1 1 , 1 2}}$ Thus, new biodegradable phosphorylated polymers for bone regeneration would be selected.

Collagen I, the major organic matrix in bone tissue, shows excellent biocompatibility and biodegradability in vivo, although the degradation of collagen scaffolds in vivo cannot be controlled, especially under the influence of collagenase. ${ }^{\mathbf{1 3}}$ However, the collagen matrix does not control the thermodynamic and kinetic mechanisms for induction of apatite nucleation on its own. The process of mineralization is mediated by non-collagenous matrix proteins (NCPs), a group of acidic, carboxylic acid and/or phosphate-containing proteins that are essential for the regulation of tissue mineralization. ${ }^{\mathbf{1 4}}$ Therefore, the surface of collagen polymer is elected to chemically alter and create mineralization nucleators with covalent linkages within the polymer network.

Alendronate is one of the common bisphosphonates that has been widely used for the treatment of various skeletal disorders, such as osteoporosis, tumour-associated osteolysis, and Paget's disease. ${ }^{\mathbf{1 5}}$ Recent studies have reported that alendronate (Aln) upregulated the expression levels of osteogenic-related genes in osteoblasts. In addition, it was reported that Aln induced the osteogenic differentiation of bone marrow-derived mesenchymal stem cells (BMSCs) and adipose-derived stem cells (ADSCs). ${ }^{16,17}$ Thus, Aln would be a potent osteoinductive molecule for use in bone tissue engineering.

In this work, we report a new type of phosphorylated polymer designed for bone tissue engineering. Collagen I is chosen as 
the backbone of the scaffold due to its biocompatibility and osteoconductivity. To improve the mineralization and osteoinductivity of the scaffold, alendronate sodium (Aln) is covalently grafted onto the collagen backbone through an aldehydeactivated reaction. Furthermore, a natural cross-linker genipin, which is approximately 10000 times less cytotoxic than glutaraldehyde, ${ }^{\mathbf{1 8 - 2 0}}$ is used as a crosslinker to modulate the crosslinking density of the phosphorylated scaffold and, thus, the stabilization. Herein, we report the synthesis and characterization of collagen I phosphorylated with Aln (Col-Aln) and its use to construct a porous scaffold. Moreover, we systematically investigate the interactions between Col-Aln and BMSCs in vitro in addition to the examination of the in vivo bone formation ability of these three-dimensional (3D) scaffolds using a clinically relevant critical-sized cranial bone defect rat model.

\section{Materials and methods}

\subsection{Materials}

The reagents used in this work were all commercially obtained from Sinopharm Chemical Reagent Beijing Co., Ltd. Alendronate sodium was purchased from Aladdin Industrial Corporation (Shanghai, China). Genipin was obtained from Yuanye Biotech Corporation (Shanghai, China).

\subsection{Synthesis of Col-Aln}

Polymers of collagen were produced by dissolving a collagen sponge in $0.5 \mathrm{M}$ of acetic acid to prepare solutions with collagen concentrations of $5 \mathrm{mg} \mathrm{mL}{ }^{-1}$. The solution was lyophilized to obtain white porous collagen. Alendronate sodium (Aln- $\mathrm{NH}_{2}$ ) was first reacted with excess glutaraldehyde in water overnight at $45{ }^{\circ} \mathrm{C}$ to obtain aldehyde-modified Aln (Aln-CHO), which was then precipitated and washed with a large amount of cold acetone. After drying, 0.09, 0.18 and $0.27 \mathrm{mg}$ of Aln-CHO were individually added into $120 \mathrm{~mL}$ of collagen solution $(5 \mathrm{mg}$ $\mathrm{mL}^{-1}$ ) and reacted overnight at room temperature. The polymers of Col-Aln were divided into three groups, Col-Aln(L), Col$\operatorname{Aln}(\mathrm{M})$, and $\mathrm{Col}-\mathrm{Aln}(\mathrm{H})$. The products were dialyzed against water for $4 \mathrm{~d}$. The solution was then lyophilized to obtain yellow porous Col-Aln. The polymers of collagen and Col-Aln were stored at $-20{ }^{\circ} \mathrm{C}$ until use.

\subsection{Scaffold characterization of Col-Aln}

FT-IR (Nicolet 6700FTIR, Thermo Fisher Scientific, USA) analysis was employed to characterize the structures of collagen, Col-Aln(L), Col-Aln(M), and Col-Aln(H). The IR spectra of the polymers were recorded at room temperature using the $\mathrm{KBr}$ pellet technique. An IR spectral range of $400-4000 \mathrm{~cm}^{-1}$ was analysed for each sample. Additionally, ${ }^{31} \mathrm{P}$ NMR spectra were recorded on a Bruker 400 NMR. X-ray photoelectron spectroscopy (XPS, ESCALAB-250Xi) was performed on an instrument equipped with a monochromatic $\mathrm{Al} \mathrm{K} \alpha \mathrm{X}$-ray source. The binding energy was calibrated with $\mathrm{C} 1 \mathrm{~s}=284.82 \mathrm{eV}$. Both N1s and $\mathrm{P} 2 \mathrm{p}$ high-resolution spectra were recorded with a pass energy of $20 \mathrm{eV}$ and an energy resolution of $0.05 \mathrm{eV}$.

\subsection{Fabrication of the 3D scaffolds}

The 3D scaffolds of collagen, Col-Aln(L), Col-Aln(M), and Col$\operatorname{Aln}(\mathrm{H})$ were cross-linked with genipin according to the method by Zhang. ${ }^{13}$ The pre-scaffolds were dissolved in $0.5 \mathrm{M}$ of acetic acid in 24-well plates and freeze-dried. Pre-scaffolds were cross-linked by immersing in $10 \mathrm{~mL}$ of PBS containing $0.5 \mathrm{wt} \%$ genipin for $48 \mathrm{~h}$ at room temperature. After being cross-linked, the scaffolds were washed thrice with deionized water to remove residual genipin and immersed in deionized water for further reaction. Afterwards, the $3 \mathrm{D}$ scaffolds were freeze-dried. The morphologies of the scaffolds were observed using a JEOL JSM6360LV scanning electron microscope.

\subsection{Release kinetics of Aln from Col-Aln scaffolds}

$100 \mathrm{mg}$ Col-Aln samples were immersed in $10 \mathrm{~mL}$ of PBS in a shaking incubator. At predetermined time intervals $(5,10,15$, 20, 25 and 30 days), a PBS sample was collected and replenished with fresh PBS. The samples were stored at $-20{ }^{\circ} \mathrm{C}$ before analysis. The concentration of Aln in all the PBS samples was determined via measuring the absorbance of the complexation of Aln and $\mathrm{Fe}(\mathrm{III})$ ions at $293 \mathrm{~nm}$ followed the previous studies. ${ }^{21}$

\subsection{In vitro mineralization in simulated body fluid}

The collagen, Col-Aln(L), Col-Aln(M), and Col-Aln(H) scaffolds were immersed in $2 \times$ simulated body fluid $(2 \times \mathrm{SBF})$ prepared according to the formula of $\mathrm{Li}^{22}$ and incubated at $37^{\circ} \mathrm{C}$ for 12 , 24,48 and 72 hours. The $2 \times$ SBF solution was replaced with fresh solution once per $12 \mathrm{~h}$. After incubation, the samples were removed, washed thrice with deionized water, freeze-dried, and examined under SEM.

\subsection{Cell isolation and culture}

Bone mesenchymal stem cells (BMSCs) were isolated from the bone marrow of Sprague-Dawley (SD) rats (4 weeks old) according to the methods reported previously. ${ }^{23}$ In brief, the femurs of the SD rats were separated under sterile conditions to expose the bone marrow cavity, which was flushed with lowglucose Dulbecco's modified Eagle's medium (LDMEM) supplemented with $10 \%$ foetal bovine serum (FBS, Life Technologies, New York, USA), 1\% penicillin and streptomycin. The released cells were collected in a $25 \mathrm{~cm}^{2}$ cell culture dish (Corning, USA) and incubated in a humidified atmosphere of $5 \% \mathrm{CO}_{2}$ at $37{ }^{\circ} \mathrm{C}$. After the cells reached $80-90 \%$ confluence, they were detached and serially sub-cultured. The BMSCs at passage 3 were used in this study.

\subsection{Cell morphology and cell proliferation}

BMSC suspensions $\left(2 \times 10^{4}\right.$ cells per $\left.\mathrm{cm}^{-2}\right)$ were cultured on collagen, Col-Aln(L), Col-Aln(M), and Col-Aln(H) (3 mm thickness) surfaces in 24-well plates in a humidified incubator. The BMSCs were washed with PBS 3 times and fixed with $4 \%$ formaldehyde solution for $30 \mathrm{~min}$ at room temperature. They were further treated with $0.1 \%$ Triton/PBS solution at $4{ }^{\circ} \mathrm{C}$ for $10 \mathrm{~min}$. After being washed with PBS 3 times, they were treated with $1 \% \mathrm{BSA} / \mathrm{PBS}$ solution for $30 \mathrm{~min}$ at room temperature to 

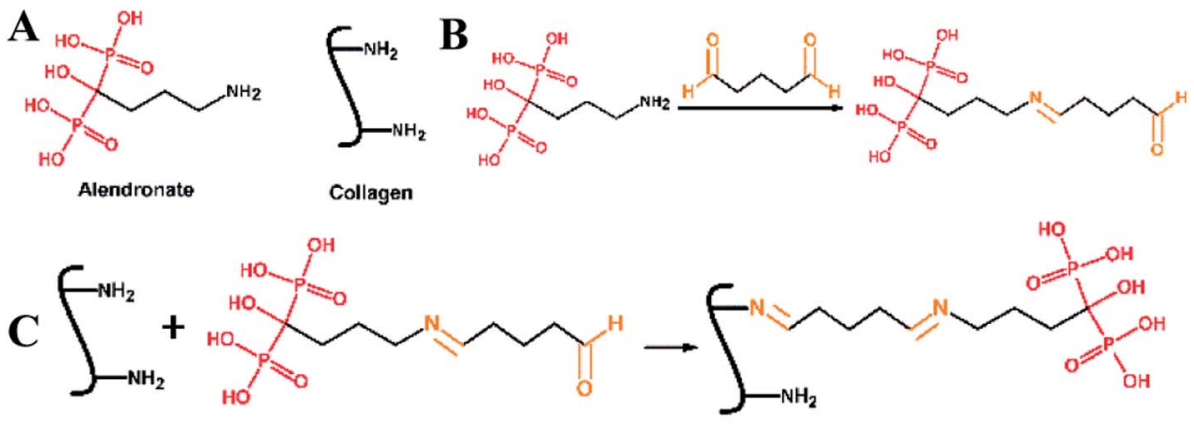

Fig. 1 Schematic illustration of the synthesis of Col-Aln 3D scaffold. (A) Structure of Aln and collagen. (B) and (C) Schematic illustration of the preparation of Col-Aln. (D) The schematic of preparation process of Col-Aln scaffolds.

block nonspecific adsorptions. Then, the cells were incubated in rhodamine phalloidin (Cytoskeleton, \#PHDR1, the $14 \mathrm{mM}$ stock solution was diluted $1: 200$ in $0.5 \% \mathrm{BSA}$ ) for $30 \mathrm{~min}$ at room temperature (in darkness). Nuclei were stained with DAPI for $1 \mathrm{~min}$ at room temperature (in darkness). Cells were then rinsed with PBS 3 times. Samples were imaged using a confocal microscope (Zeiss, LSM 710).

The proliferation of BMSCs was measured using the Cell Counting Kit-8 (CCK-8, Dojindo). Cells were cultured on collagen, $\mathrm{Col}-\mathrm{Aln}(\mathrm{L})$, Col-Aln(M), and $\mathrm{Col}-\mathrm{Aln}(\mathrm{H})$ surfaces as

A
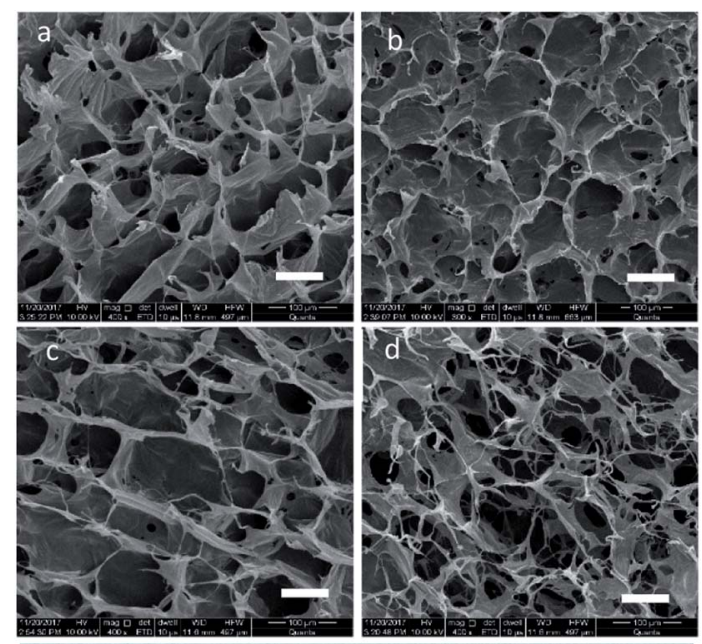

B

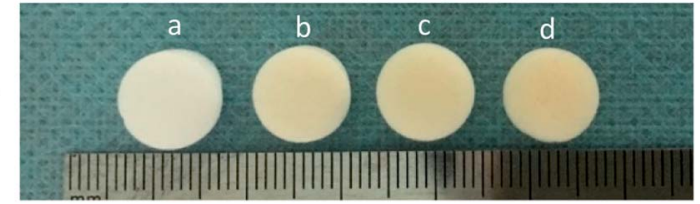

Fig. 2 (A) SEM images and (B) gross images of (a) collagen, (b) Col$A \ln (\mathrm{L})$, (c) Col-Aln(M), and (d) Col-Aln(H) scaffolds, 400x, scale bar = $100 \mu \mathrm{m}$ All scaffolds had connected a porous structure, and the pore size ranged from 100 to $250 \mu \mathrm{m}$. above for $0,1,3,5$ days in DMEM supplemented with $10 \%$ foetal bovine serum and $1 \%$ penicillin-streptomycin liquid $(100 \mathrm{U}$ $\mathrm{mL}^{-1}$ ) and then incubated in $10 \%$ CCK-8 solution in a $5 \% \mathrm{CO}_{2}$ incubator at $37{ }^{\circ} \mathrm{C}$ for $2 \mathrm{~h}$. The absorbance of the culture medium was then measured at $450 \mathrm{~nm}$.

\subsection{Gene and protein expression of osteogenic differentiation}

The expression of bone-related genes Runx-2 and ALP by BMSCs on collagen, Col-Aln(L), Col-Aln(M), and Col-Aln(H) scaffolds was evaluated after 7 and 14 days of culturing using the reversetranscription polymerase chain reaction (RT-PCR). The total RNA of both groups was extracted using TRIzol reagent (Invitrogen Inc., Carlsbad, CA, USA) for RT-qPCR detection. PCR was performed using the Transcriptor cDNA Synth Kit and FastStart Universal SYBR Green Master (Roche) following the manufacturer's instructions. The relative level of expression of each target gene was then calculated using the $2^{-\Delta \Delta C_{\mathrm{t}}}$ method. RT-qPCR primers were designed based on cDNA sequences from the NCBI Sequence database, using the primer pairs: Runx-2 (F-5'-AGTGTCATCATCTGAAATACGC- $3^{\prime}, \quad$ R-5'-CACCAAGTCCT-TTTAATCCAC- $3^{\prime}$ ), ALP(F-5'-CTTTCCCATCTTCCGACACTG-3' ${ }^{\prime}$, R-5'-CTGGCGACATGATACTGGCTAT- $3^{\prime}$ ), and GAPDH (F-5'-TCTCTGCTCCTCCCTGTTC$3^{\prime}$, R-5'-ACACCGACCTTCACCATCT- $3^{\prime}$ ).

After culturing for 14 days on different scaffolds, the BMSCs were harvested, washed with PBS three times and completely homogenized in radio immunoprecipitation assay buffer (RIPA) with protease inhibitors. Then, $15 \mu \mathrm{g}$ of protein from each sample was separated on SDS-PAGE gels and then transferred onto a polyvinylidene fluoride (PVDF) membrane (Millipore, MA, USA). After being blocked with blocking buffer for $1 \mathrm{~h}$, the membranes were incubated with primary antibodies against ALP (1:1000, Abcam, USA), Runx2 (1:1000, Abcam, USA), and GAPDH (1:2000, Abcam, USA) overnight at $4{ }^{\circ} \mathrm{C}$. The membranes were washed three times in TBST buffer, 

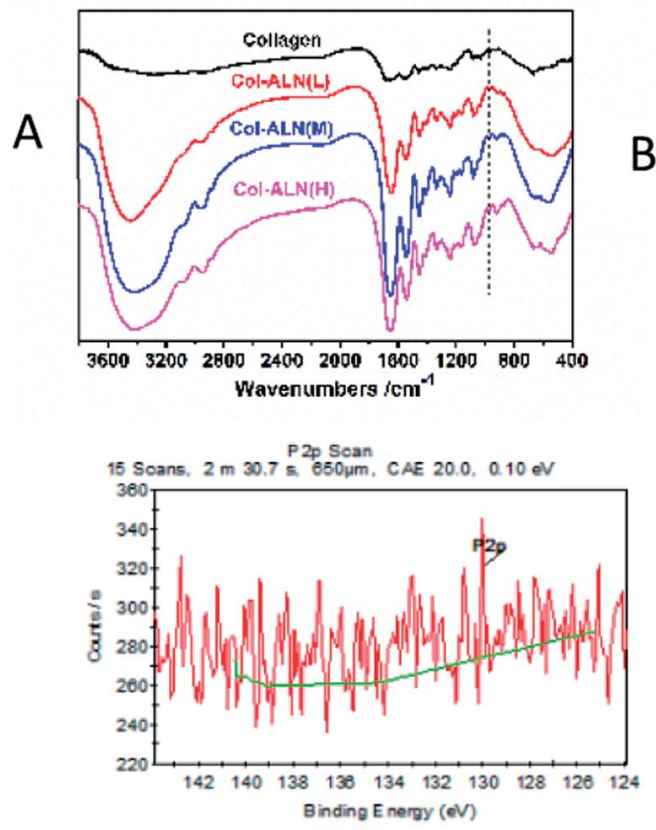

Collagen

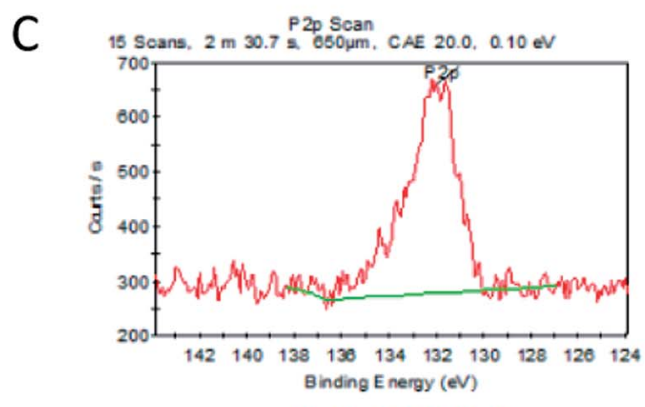

Col-ALN(M)
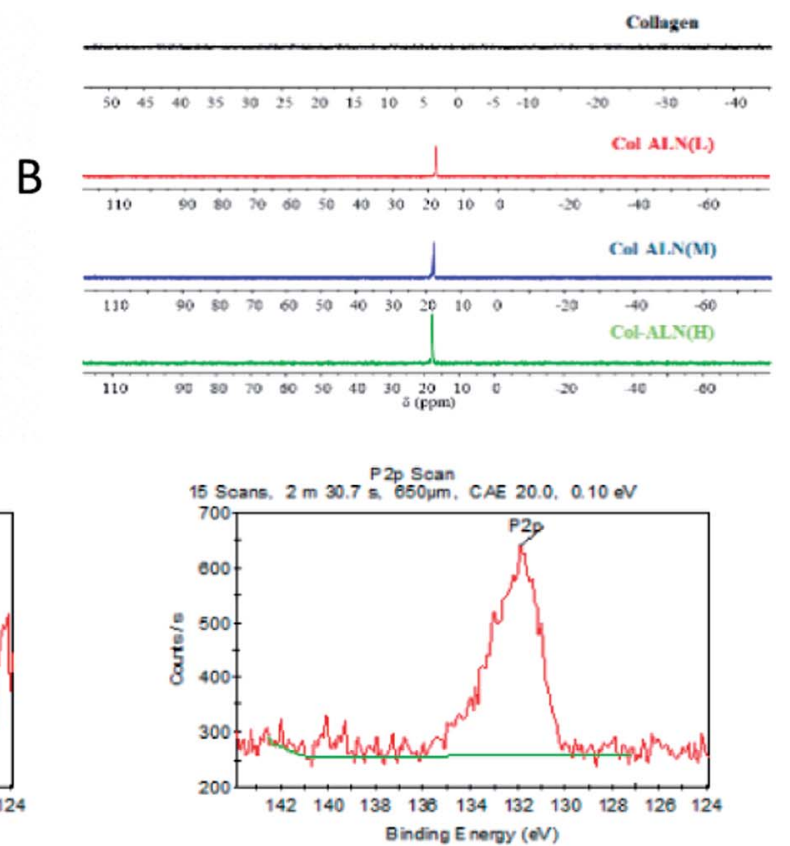

Col-ALN(L)

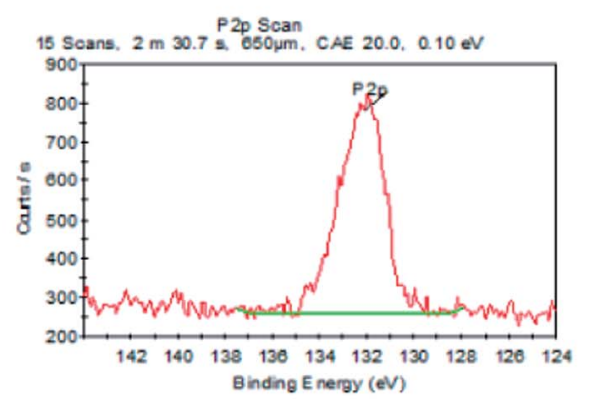

Col-ALN(H)

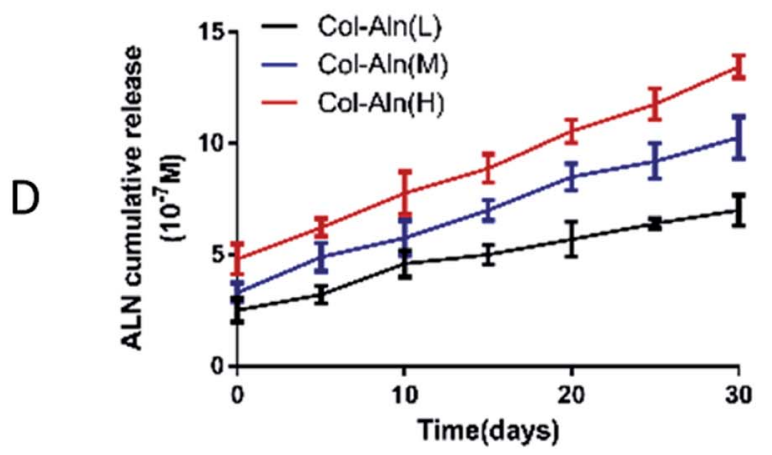

Fig. 3 (A) FTIR spectra of different scaffolds. A new peak appeared at the absorbance of $940 \mathrm{~cm}^{-1}$, which was assigned to the stretching vibration of $\mathrm{P}-\mathrm{O}(\mathrm{B}){ }^{31} \mathrm{P}$ NMR spectra of different scaffolds. Only the Col-Aln had an obvious resonance peak. (C) X-ray photoelectron spectroscopy of different scaffolds (D) In vitro cumulative release of Aln from Col-Aln scaffolds.

incubated with anti-rabbit HRP-conjugated secondary antibodies at a $1: 4000$ dilution for $1 \mathrm{~h}$ at room temperature and then detected using an enhanced chemiluminescence (ECL Western Blotting Substrate, Pierce, USA) system. The relative intensities of the protein bands were quantified using ImageJ software (National Institutes of Health, Bethesda, Maryland, USA).

\subsection{In vivo calvarial defect repair experiment}

All animal procedures were performed following a protocol approved by Animal Ethical and Welfare Committee of Sun YatSen University (IACUC-DB-17-1104). Eighteen male 250-260 g $\mathrm{SD}$ rats were utilized for this experiment. Under general anaesthesia, the cranium was exposed through a medial incision. Bilateral full-thickness circular defects $(5 \mathrm{~mm}$ in diameter, 

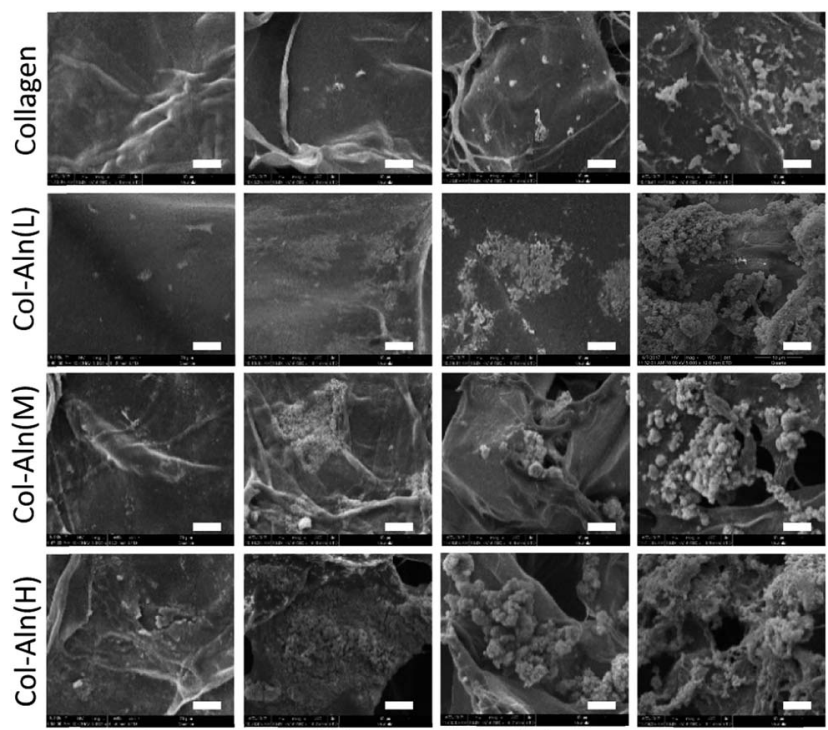

(b)

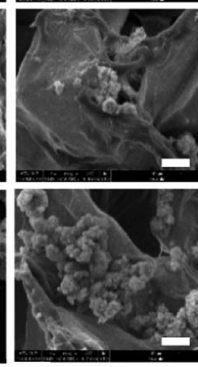

(c)

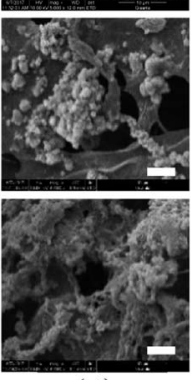

(d)

Fig. 4 SEM images of biomimetic mineralization in $2 \times$ SBF after (a) $12 \mathrm{~h}$, (b) $24 \mathrm{~h}$, (c) $48 \mathrm{~h}$, (d) $72 \mathrm{~h}, 5000 \times$, scale bar $=10 \mu \mathrm{m}$. The amount and velocity of mineralization increased with time and the Aln content of the collagen scaffolds.

$1 \mathrm{~mm}$ in thickness) were created using a trephine. The size of the bilateral calvarial defects used in the present study was determined according to the protocol of previously reported studies. $^{24,25}$ Collagen and Col-Aln(H) scaffolds were implanted into the defects. The blank groups were left untreated. After placement of the scaffolds, the soft tissues were closed with sutures. Each rat was then passed back to the veterinary surgeon for routine post-operative care. The whole calvarias were harvested for evaluation at the predetermined times of 4 weeks and 8 weeks.

\subsection{Evaluation of bone regeneration in vivo}

The specimens harvested at both 4 and 8 weeks were immediately fixed in $4 \%(\mathrm{v} / \mathrm{v})$ paraformaldehyde for $24 \mathrm{~h}$. Microcomputed tomography $(\mu \mathrm{CT})$ scans were carried out on each specimen to quantify new bone formation within the calvarial defect. The Scanco $\mu 50$ Micro-CT scanning device (Scanco Medical, Bassersdorf, Switzerland) was set to $70 \mathrm{kVp}, 200 \mathrm{~mA}$, $300 \mathrm{~ms}$ integration time and an isotropic voxel size of $10 \mu \mathrm{m}$ for detailed evaluation.

The fixed sample specimens were decalcified by submersion in $10 \%$ EDTA ( $\mathrm{pH} 7.0)$ for a month at $37^{\circ} \mathrm{C}$ on a rotating rocker. After being embedded in paraffin wax, the specimens were serially dehydrated in a graded series of ethanol washes. Then, 5 mm-thick sections were made and stained according to the standard protocols for H\&E and Trichrome-Masson staining. Images from each specimen were acquired and digitized using transmitted light and epifluorescence microscopic visualization (ZEISS Axio Image.Z2, Germany). Quantitative analysis was performed on sections from each specimen, and a single image of each section was acquired and digitized. The development of new bone was quantified with a computer-based image analysis system (Image-Pro Plus 4.0, Media Cybernetics, Silver Spring,
MD, USA). The percentage of new bone (NB (\%)) was expressed as the ratio of the neoformative bone area to the original total defect area. ${ }^{26}$

\subsection{Statistical analysis}

All of the data were presented as the means \pm standard deviation (SD), and $P<0.05$ was considered significant. One-way ANOVA was applied to compare different groups using SPSS software (IBM SPSS, Armonk, New York, USA).

\section{Results}

\subsection{Structure and characterization of collagen and Col-Aln scaffolds}

We created a new phosphorylated scaffold (Fig. 1), Col-Aln, that combines the osteoconductive backbone of collagen with the osteoinductive molecule Aln through an aldehyde-activated reaction, and crosslinked by genipin into 3D scaffolds eventually. Fig. 2 shows the structure and gross images of the collagen, Col-Aln(L), Col-Aln(M), and Col-Aln(H) scaffolds synthesized by the freeze-drying method. The SEM images of all of the scaffolds showed that the scaffolds were comprised of a porous structure suitable for bone tissue regeneration. All scaffolds had connected a porous structure, and the pore size ranged from 100 to $250 \mu \mathrm{m}$. Comparing the FTIR spectra (Fig. 3a) of the collagen, Col-Aln(L), Col-Aln(M), and Col-Aln(H), it was seen that in the spectra of Col-Aln(L), Col-Aln(M), and Col-Aln(H), a new peak appeared at the absorbance of $940 \mathrm{~cm}^{-1}$, which was assigned to the stretching vibration of $\mathrm{P}-\mathrm{O}$. An additional peak at the absorbance of $1560 \mathrm{~cm}^{-1}$ was assigned to the stretching vibration of amide II. The ${ }^{31} \mathrm{P}$ NMR spectra (Fig. 3b) revealed that only the Col-Aln had an obvious resonance peak at $17.6 \mathrm{ppm}$. Differences in the P2p spectra were observed for different scaffolds (Fig. 3C). As expected, no obvious peaks were detected in the collagen scaffold, indicating the absence of a phosphorus element. For Col-Aln(L), Col-Aln(M), and Col-Aln(H), the P2p spectra all presented a P2p peak structure, which could imply various chemical states of phosphorus. The increase of the P2p peak intensity from Col-Aln(L) to Col-Aln(H) demonstrated the increasing amount of Aln in the scaffolds. These results suggested the successful incorporation of Aln into the collagen scaffolds.

To determine the release kinetics of Aln from Col-Aln scaffolds, Aln concentration was spectrophotometrically determined via complexation with Fe(III) ions in all samples. Three gradient concentrations of Aln (Col-Aln(L), Col-Aln(M), and Col$\operatorname{Aln}(\mathrm{H})$ ) were combined. Results showed that Aln showed sustained release for more than 30 Days in all groups, without a burst or stagnant release (Fig. 3D).

\subsection{Biomimetic mineralization of 3D scaffolds}

The collagen, Col-Aln(L), Col-Aln(M), and Col-Aln(H) scaffolds were cultured in $2 \times \mathrm{SBF}$ to test their capacity for mineralization in vitro. The SEM images (Fig. 4) showed a surface morphology of mineralized scaffolds. At the $12 \mathrm{~h}$ observation point, there were hardly any particles found on the surface of the collagen, 

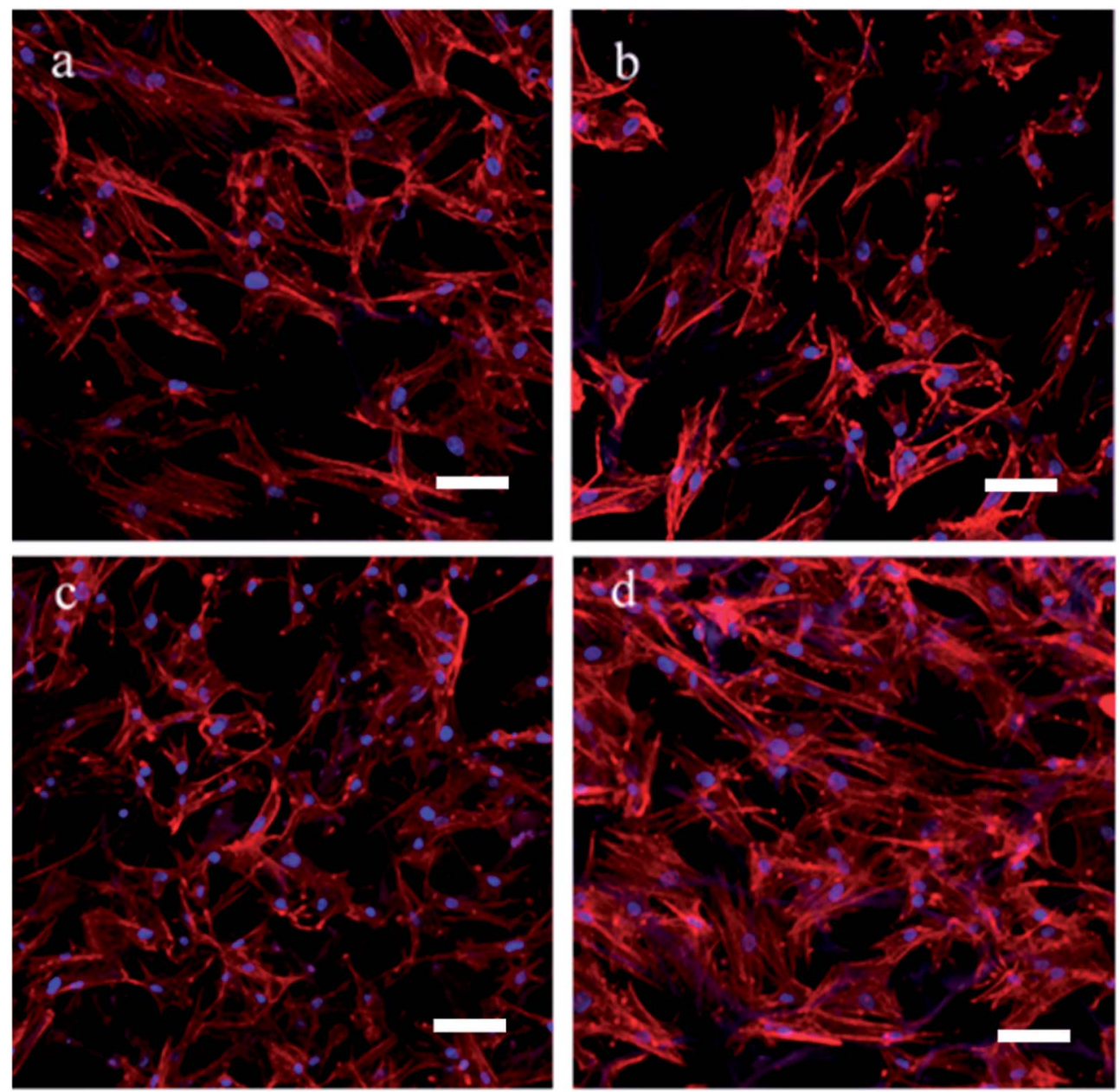

Fig. 5 Cell morphology of BMSCs being cultured on (a) collagen, (b) Col-Aln(L), (c) Col-Aln(M), (d) Col-Aln(H) scaffold for 7 d by CLSM, respectively. Scale bar $=100 \mu \mathrm{m}$.

and few particles appeared on the Col-Aln scaffolds. The amount and velocity of mineralization increased with time and the Aln content of the collagen scaffolds. After $72 \mathrm{~h}$, heavy mineralization could be found on the $\operatorname{Col}-\mathrm{Aln}(\mathrm{M})$ and Col$\operatorname{Aln}(\mathrm{H})$ scaffolds.

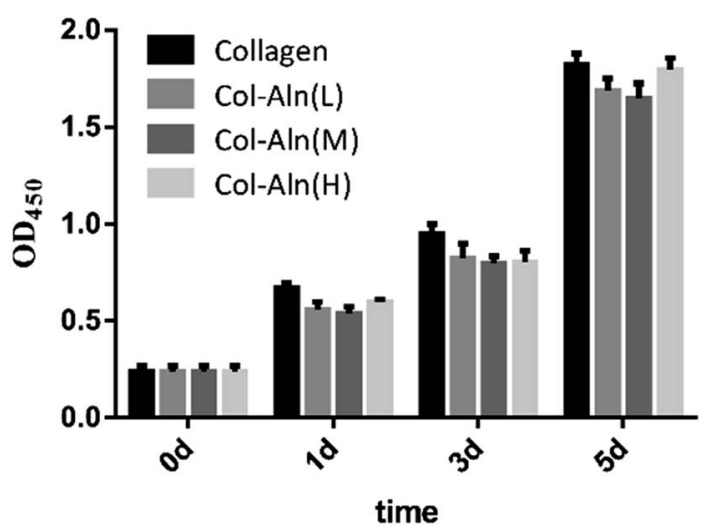

Fig. 6 Effects of different scaffolds on proliferation in BMSCs by cck-8 test. $* P<0.05$. There was no significant difference between the levels of cell proliferation on the different scaffolds at the various observation times.

\subsection{Morphology and proliferation of BMSCs on scaffolds}

To determine the effects of the scaffolds on cell growth, the BMSCs were cultured on all of the scaffolds for $7 \mathrm{~d}$. Confocal images indicated that all of the BMSCs showed a similar spindle morphology, suggesting that all scaffolds had good cytocompatibility (Fig. 5a-d). A large amount of BMSCs was found on the scaffolds with or without Aln, likely due to the good biocompatibility of the scaffolds. Cell proliferation was measured using a CCK-8 assay (Fig. 6), and the cell number significantly increased in all groups. In addition, there was no significant difference between the levels of cell proliferation on the different scaffolds at the various observation times, which suggested that alendronate-modified collagen scaffolds did not negatively influence the cytocompatibility.

\subsection{Effects of the Col-Aln on the osteogenic differentiation of BMSCs}

The ability of collagen, Col-Aln(L), Col-Aln(M), and Col-Aln(H) scaffolds to facilitate the osteogenic differentiation of BMSCs was investigated by examining the expression of Runx-2 and ALP genes, which code for bone-specific cytokines, and these results were normalized using GAPDH as a house-keeping gene 

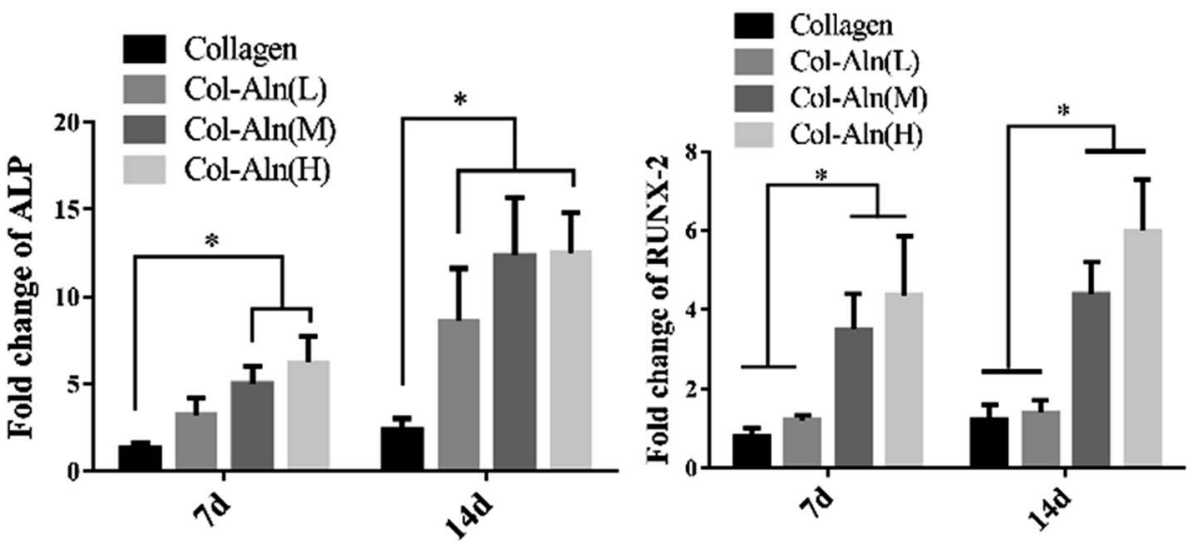

Fig. 7 The level of the mRNA for osteogenic-specific genes ALP and RUNX-2 of BMSCs cultured on different scaffolds for 7 and 14 days. Levels, quantified using RT-PCR. $\left({ }^{*} p<0.05\right)$.

(Fig. 7). All expressions of the tested genes were higher for the Col-Aln(M) and Col-Aln(H) groups than for the collagen groups at 7 and 14 days. The ALP expression levels of the Col-Aln(L) groups was markedly more upregulated at $14 \mathrm{~d}$ than collagen groups $(p<0.05)$. With the exception of no significant difference in Runx-2 gene expression between the Col-Aln $(\mathrm{L})$ and collagen groups after 14 days $(p>0.05)$. There was no significant difference between the abilities of $\mathrm{Col}-\mathrm{Aln}(\mathrm{M})$ and $\mathrm{Col}-\mathrm{Aln}(\mathrm{H})$ scaffolds to promote the osteogenic differentiation of BMSCs after 14 days.

To further examine the effect of the Col-Aln scaffolds on the osteogenic differentiation of BMSCs, we examined the osteogenic expression of ALP and Runx-2 proteins. Western blot analysis showed that the expression of ALP and Runx-2 proteins was significantly upregulated in response to all of the Col-Aln

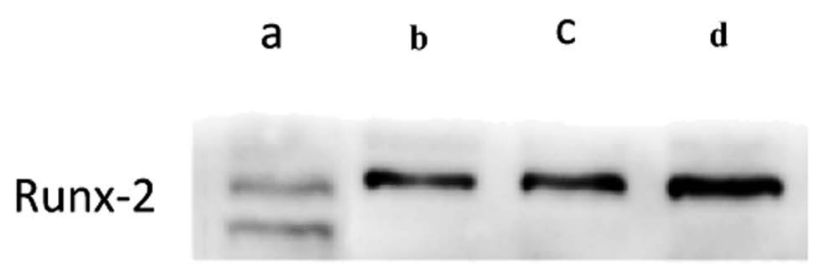

ALP
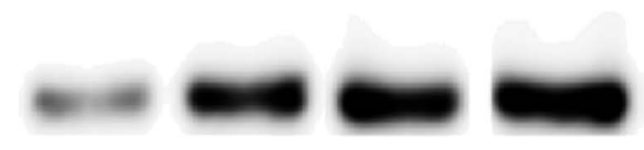

GAPDH

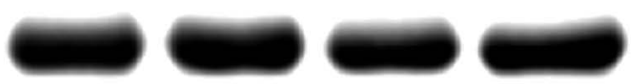

scaffolds after 14 days of culture, especially with the Col$\operatorname{Aln}(\mathrm{H})$ scaffold $(p<0.05)$ (Fig. 8).

Taken together, these results demonstrated that the Col-Aln scaffolds could promote osteogenesis in vitro, and Col-Aln $(\mathrm{H})$ seemed to be the most beneficial to the osteogenic differentiation of BMSCs. Therefore, we chose the Col-Aln(H) scaffold for the next experiment in vivo.

\subsection{Evaluation of bone formation in the critical-defect animal model by micro-CT analysis}

To observe the ability of Col-Aln to facilitate osteogenesis, scaffolds of collagen and $\mathrm{Col}-\mathrm{Aln}(\mathrm{H})$ were implanted in the critical defect of SD rats. After 4 and 8 weeks $(n=6)$, the entire calvarial bone was extracted and evaluated by micro-CT analysis. As shown in Fig. 9A, the defects in the collagen and Col- 

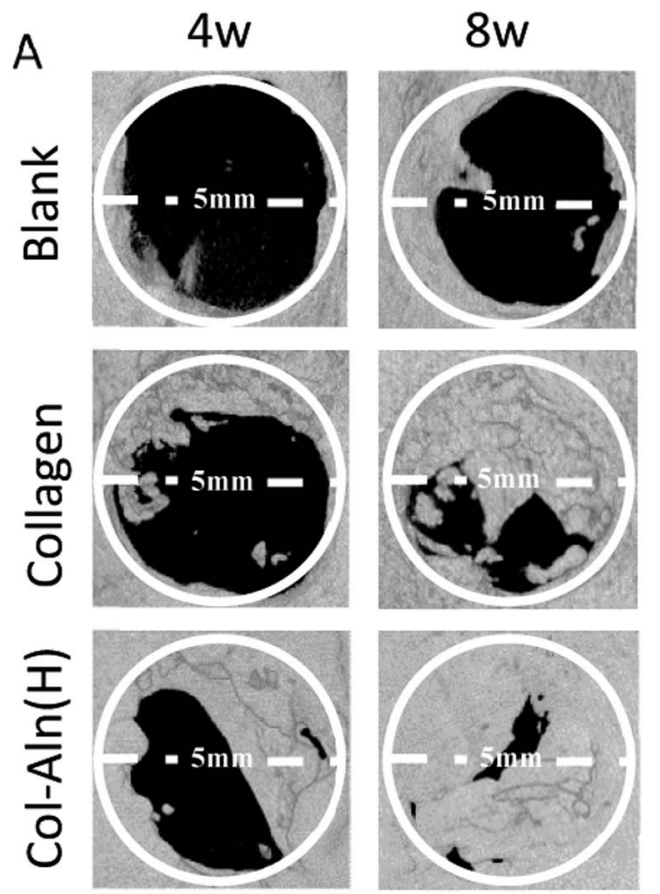

\section{$8 w$}
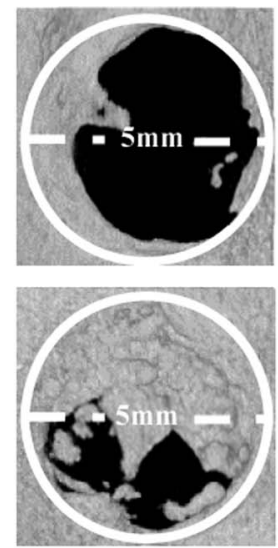

B

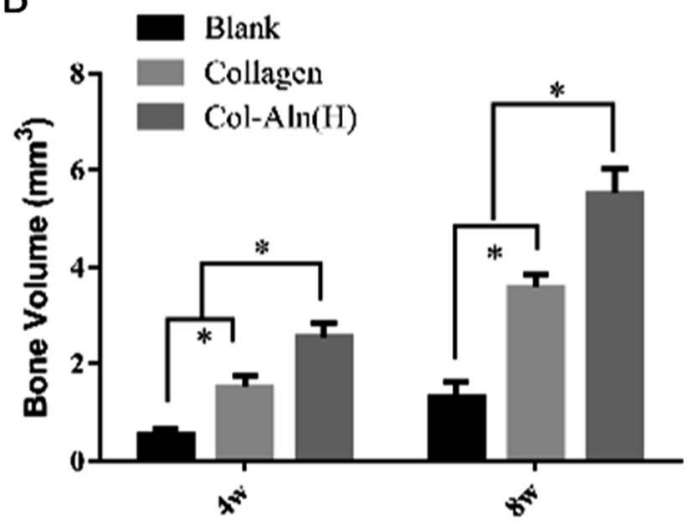

Fig. 9 (A) 3D micro-CT reconstructed images at 4 and 8 weeks with different implants. (B) Micro-CT examination of the bone volume of whole calvarias after 4 and 8 weeks implantation in vivo. ${ }^{*} p<0.05$.

$\operatorname{Aln}(\mathrm{H})$ groups were healed to different levels with new bone tissue in growth in the scaffolds, whereas the rates of bone formation were considerably slower in defects in the blank group. Quantification of the newly formed bone within the defect confirmed the above observations (Fig. 9B). The bone volume in the defects was significantly higher in the Col-Aln $(\mathrm{H})$ group than in the other two groups at both 4 and 8 weeks. Micro-CT measurements demonstrated that the phosphorylated scaffolds promoted significantly more bone formation than did the unmodified collagen scaffolds.

\subsection{Histological observation}

We also examined bone tissue regeneration in the implants histologically. In Fig. 10, characteristic images from the three groups are presented. Histological analysis of decalcified tissue sections using H\&E and Trichrome-Masson staining was conducted at the predetermined time after operation. The histological examination revealed the formation of regenerated bone with a structure typical of mature bone in the central part of the repaired area in the Col-Aln $(\mathrm{H})$ group after 4 weeks postimplantation. In contrast, no obvious bone was present in the blank group, but fibrous tissue was detected. For 8 weeks postimplantation, the remaining scaffold materials were detectable in the collagen and Col-Aln $(\mathrm{H})$ groups. In contrast with the lack of typical bone formation in the defect area of the blank group, a large amount of tissue formation was found in the repaired area in the collagen and Col-Aln $(\mathrm{H})$ groups. Particularly, larger bones were present in the group implanted with $\operatorname{Col}-\mathrm{Aln}(\mathrm{H})$.

The quantitative estimation of the new bone percentage (NB (\%)) was calculated using the ratio of the neoformative bone area to the original total defect area. As the results in Fig. 11 show, as the implantation time increased from 4 weeks to 8 weeks, the NB (\%) increased from $15.5 \pm 2.8 \%$ to $28.7 \pm 4.5 \%$ in defects treated with collagen. In comparison, bone formation was superior in Col-Aln(H)-treated defects throughout the whole evaluation period, during which the mean NB (\%) values increased from $22.2 \pm 1.7 \%$ to $43.5 \pm 5.4 \%$ at the corresponding times. The results demonstrated that the phosphorylated scaffold of Col-Aln with its excellent osteoinduction properties would be a promising candidate as a bone substitute in the repair of cranial defects.

\section{Discussion}

The objective of this study was to develop a phosphorylated scaffold with properties that could induce fast mineralization and to provide an analysis of osteogenic potential both in vitro and in vivo. The initial stage of in vivo biomineralization, such as the nucleation of vertebrate teeth and bone mineralization, ${ }^{\mathbf{9 1 4}}$ occurs through the interaction of immobilized, negatively charged functional groups with calcium and phosphate ions. ${ }^{27}$ Col-Aln, consisting of collagen covalently bonded with varying amounts of phosphate-containing alendronate, can be used to create a scaffold that contains a permanent negative charge for mineral nucleation. ${ }^{28}$

Our data demonstrated that various amounts of Aln could be incorporated into the collagen scaffold, and incorporation of Aln did not have an adverse effect on the biocompatibility of collagen. An increase in mineralization deposition on the scaffolds was observed with increasing Aln concentrations. Since phosphate groups are negatively charged under 

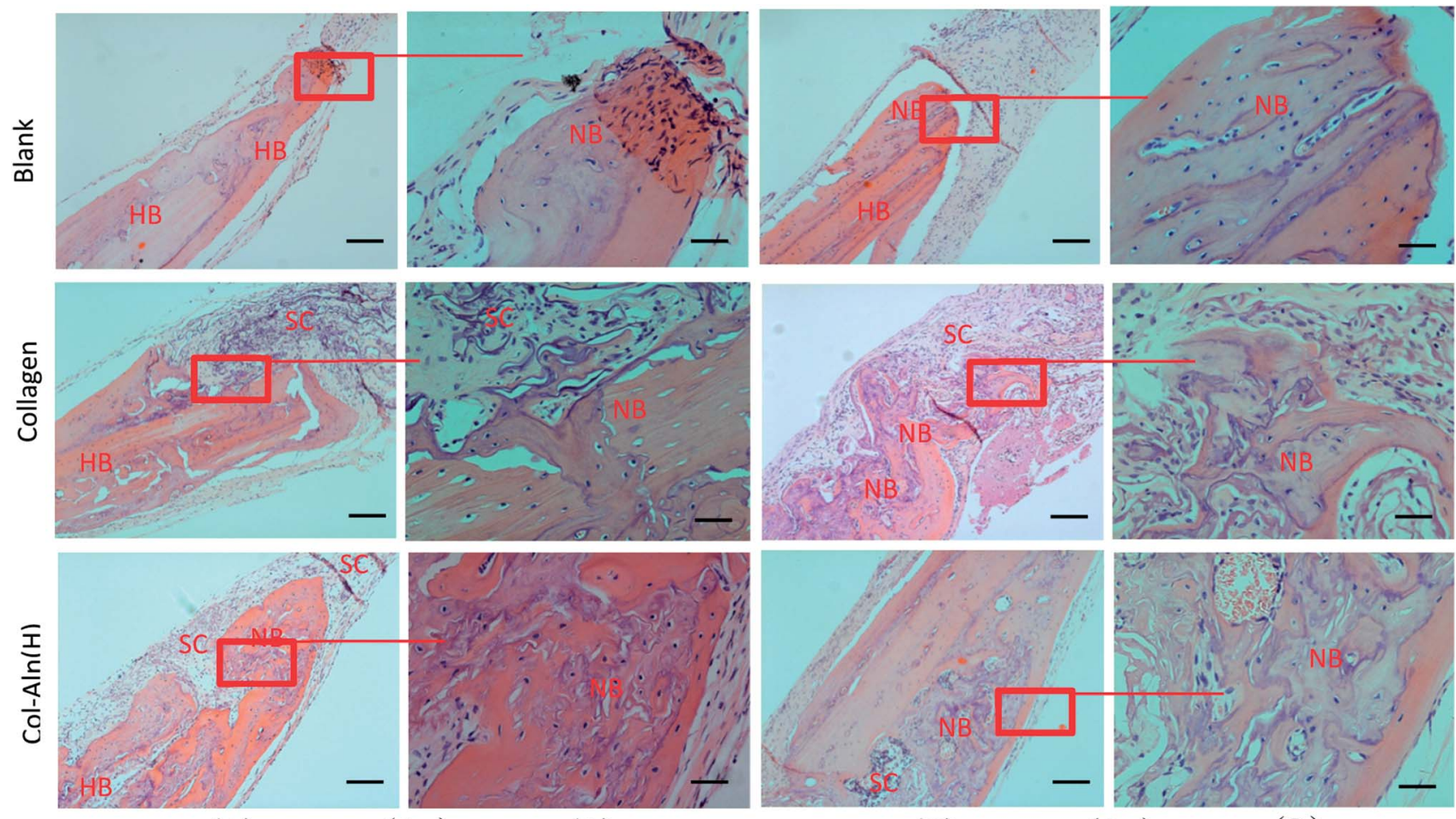

(A)

(4w)

(B)
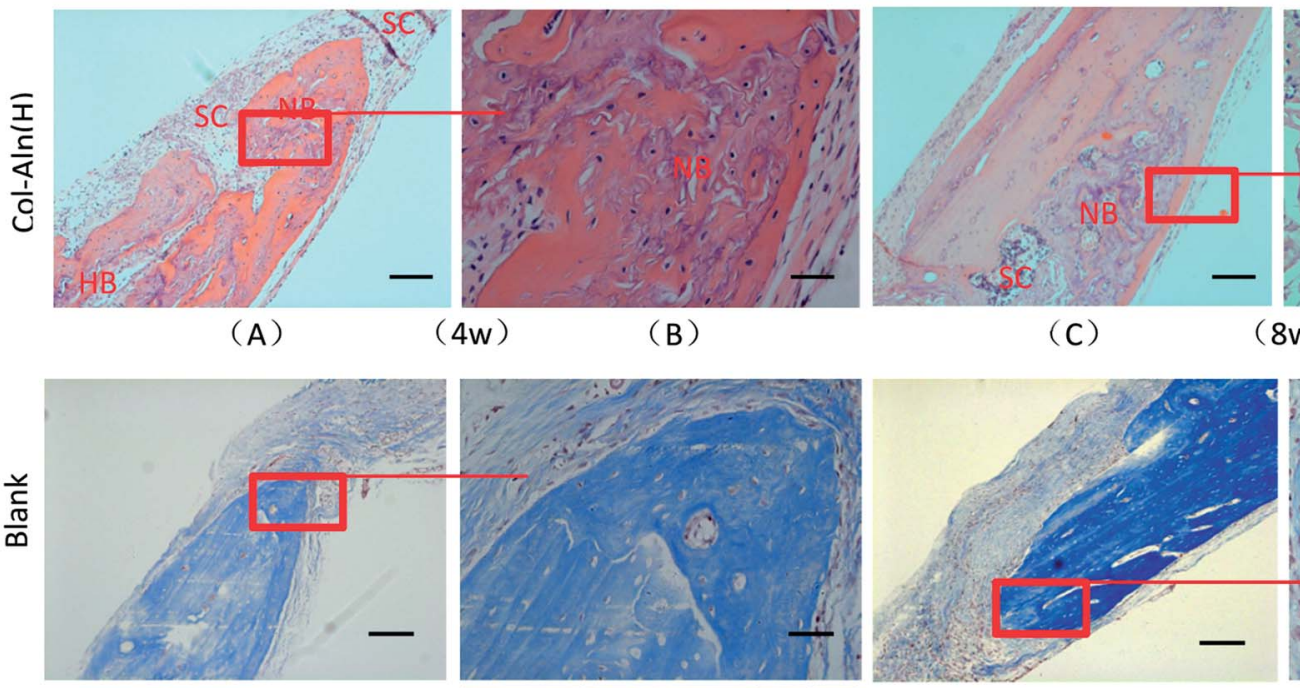

(8w)

(D)
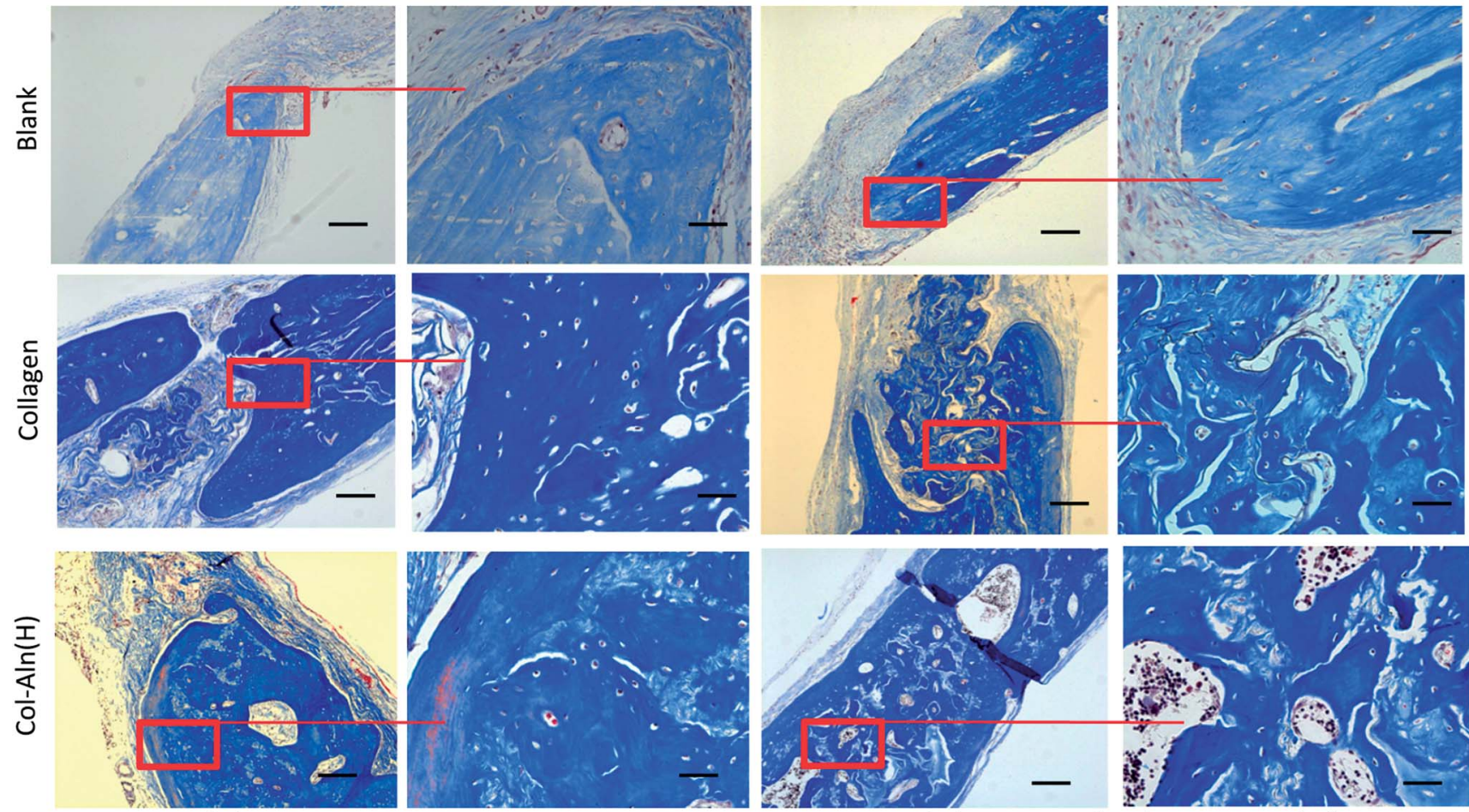

(E)

(4w)

(F)

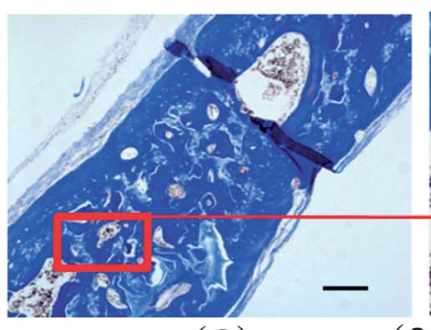

(G)

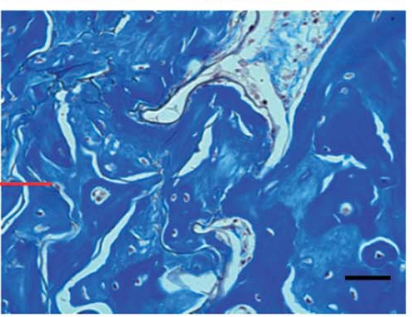

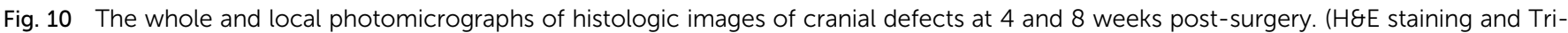
chrome-Masson staining) abbreviations and signs used: host bone (HB), new bone (NB), scaffold (SC). The bar represents $250 \mathrm{~mm}$ in A/C/E/G and $100 \mathrm{~mm}$ in $\mathrm{B} / \mathrm{D} / \mathrm{F} / \mathrm{H}$.

physiological conditions, their negative charge has been suggested to promote the chelation of calcium ions from solution, which can initiate the mineralization process. ${ }^{29}$
The bioactivity of Col-Aln could be ascribed to its abundance of phosphate groups, which have been demonstrated to be key bioactive moieties in phosphorylated scaffolds. ${ }^{30}$ Previous 


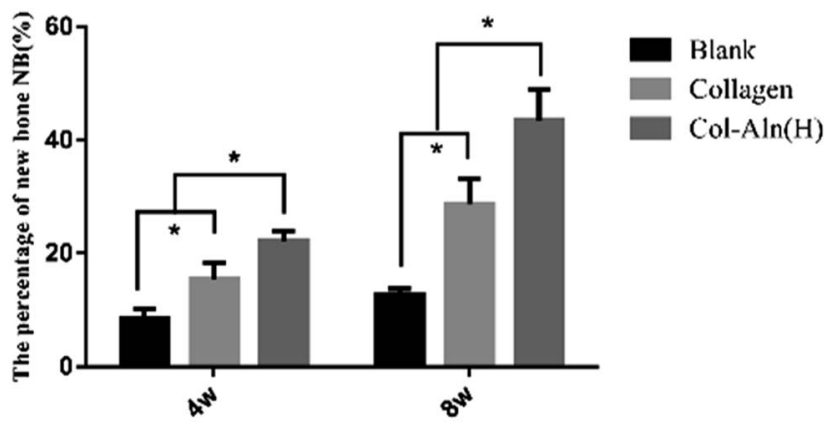

Fig. 11 The percentage of new bone (NB (\%)) was expressed as the ratio of the neoformative bone area to the original total defect $* p<$ 0.05 .

studies $^{22,31}$ showed that biomaterials with phosphate groups facilitated faster and more complete mineralization than did analogues lacking phosphorous-containing groups. Furthermore, phosphate groups provide an ideal environment for osteogenesis to produce corresponding matrix proteins, such as Runx-2 and ALP (Fig. 7), which further induce ossification. Additionally, the negative charge has been shown to inhibit the adsorption of nonspecific proteins, such as albumin, because these proteins are also negatively charged at physiological pH. ${ }^{32,33}$ Furthermore, negatively charged surfaces have been shown to specifically adsorb cell-adhesive proteins such as fibronectin for improved cell adhesion. ${ }^{30,33}$ More bone has been shown to form on negatively charged ceramic surfaces as well. ${ }^{34}$ Thus, the negative charges of phosphate groups may also contribute to the enhancement of the function and differentiation in BMSCs.

The in vivo study set out to evaluate the ability of both ColAln and original collagen scaffolds to facilitate bone growth within a critical-sized rat cranial defect, as well as to assess the ability of hydrogels to undergo in vivo degradation. Bone volume within the defect after 4 and 8 weeks measured in the present study demonstrated that the higher levels of Col-Aln resulted in significantly more new bone in the defect from the surrounding the centre, as indicated by both histological and $\mu \mathrm{CT}$ analyses. The results observed with higher levels of Aln could indicate that the increased phosphate content could have guided bone growth across the hydrogel surface. ${ }^{35}$ Bone tissue infiltrating into the scaffold indicated that these scaffolds have the potential to integrate with host tissue and accelerate bone formation. These 3D porous structures are able to maintain their configuration, permitting easy cell infiltration into the scaffold structure, which avoided a lack of mineralization near the centre of the hydrogel materials. ${ }^{36,37}$

In addition to the phosphate groups, one of the key design features of Col-Aln is the integration of Aln, a widely used antiosteoporosis molecule, which is able to promote osteogenic differentiation. Compared to the other macromers used for phosphorylated scaffolds, Aln, as a small molecule, was expected to increase the content of phosphate groups in the scaffold. Additionally, after degradation, Aln can work continuously to promote osteogenic differentiation of BMSCs via several mitogen-activated protein kinase (MAPK) pathways, such as in extracellular signal-related kinase (ERKs) $1 / 2$ and Jun amino-terminal kinase (JNK1/2/3) pathways, in a dosedependent manner. ${ }^{38}$ When Aln molecules are in solution, they are readily taken up by osteoclasts and inhibit bone resorption by signalling apoptosis in these osteoclasts. ${ }^{39,40}$

Another major strength of the scaffolds in this study was their ability to undergo in vivo degradation. Both collagen and Col-Aln scaffolds demonstrated degradation within the defect. Degradability is vital for tissue engineering applications, as it allows for tissue ingrowth into the scaffold and eventual replacement of the scaffold with host tissues. ${ }^{10}$ Histological examination of the sections revealed that most of the Col-Aln and collagen scaffolds had degraded after 8 weeks, and bone tissue and blood vessels had infiltrated into the scaffolds, demonstrating the osteogenic potential of these scaffolds. Last, but not least, phosphorylation of the scaffolds completely prevented fibrous capsule formation and promoted mineral deposition around the Col-Aln implants in vivo. This result is a significant improvement over those presented in previous reports. ${ }^{32,41}$

\section{Conclusions}

We created a new phosphorylated scaffold, Col-Aln, that combines the osteoconductive backbone of collagen with the osteoinductive molecule Aln. Col-Aln showed excellent mineralization in SBF and compatibility with BMSCs in vitro. To the best of our knowledge, this is the first time that a bioactive phosphorylated 3D scaffold for bone regeneration has been produced based on the small molecule Aln covalently grafted onto the collagen. Our results revealed that BMSCs are able to attach and undergo osteogenic differentiation without the use of external osteogenic factors. These findings suggest that the presence of phosphate on a bone cell attachment surface is an important factor in the subsequent behaviour of the cells that anchor onto that surface. Furthermore, the Col-Aln scaffold was shown to readily degrade within the defect, allowing for the infiltration of host tissue and bone formation within the Col-Aln scaffold. Thus, the phosphorylated Col-Aln scaffold described in this study is an attractive candidate for bone tissue engineering applications.

\section{Conflicts of interest}

There are no conflicts of interest to declare.

\section{Acknowledgements}

Y. C. H. and T. Z. contributed equally to this work. The authors declare no conflicts of interest in this work. The authors gratefully acknowledge the funding from the National Nature Science Foundation of China (51502095 \& 81670984), the International Cooperation Project of Science and Technology in Guangdong Province (Grant No. 2016B050502008), and the Guangdong Natural Science Funds for Distinguished Young Scholars (2016A030306018). 


\section{References}

1 D. H. Lee, N. Tripathy, J. H. Shin, J. E. Song, J. G. Cha, K. D. Min, C. H. Park and G. Khang, Int. J. Biol. Macromol., 2017, 95, 14-23.

2 N. Cui, J. Qian, J. Wang, C. Ji, W. Xu and H. Wang, Mater. Sci. Eng., C, 2017, 71, 118-124.

3 Y. Dan, O. Liu, Y. Liu, Y. Y. Zhang, S. Li, X. B. Feng, Z. W. Shao, C. Yang, S. H. Yang and J. B. Hong, Nanoscale Res. Lett., 2016, 11, 487.

4 Y. Fillingham and J. Jacobs, Bone Joint J., 2016, 98-B, 6-9.

5 Y. Ding, Y. Su, Z. Lv, H. Sun, X. Bi, L. Lu, H. Zhou, Z. You, Y. Wang, J. Ruan, P. Gu and X. Fan, Mater. Sci. Eng. C, 2017, 76, 249-259.

6 P. Poh, D. W. Hutmacher, B. M. Holzapfel, A. K. Solanki, M. M. Stevens and M. A. Woodruff, Acta Biomater., 2016, 30, 319-333.

7 P. Datta, J. Chatterjee and S. Dhara, J. Biomater. Sci., Polym. Ed., 2013, 24, 696-713.

8 P. Huang, X. Bi, J. Gao, L. Sun, S. Wang, S. Chen, X. Fan, Z. You and Y. Wang, J. Mater. Chem. B, 2016, 4, 29-211.

9 L. S. Gu, Y. K. Kim, Y. Liu, K. Takahashi, S. Arun, C. E. Wimmer, R. Osorio, J. Q. Ling, S. W. Looney, D. H. Pashley and F. R. Tay, Acta Biomater., 2011, 7, 268-277.

10 B. M. Watson, F. K. Kasper, P. S. Engel and A. G. Mikos, Biomacromolecules, 2014, 15, 1788-1796.

11 P. Datta, J. Chatterjee and S. Dhara, Colloids Surf., B, 2012, 94, 177-183.

12 D. S. W. Benoit, M. P. Schwartz, A. R. Durney and K. S. Anseth, Nat. Mater., 2008, 7, 816.

13 X. Zhang, X. Chen, T. Yang, N. Zhang, L. Dong, S. Ma, X. Liu, M. Zhou and B. Li, Cell Tissue Banking, 2014, 15, 531-541.

14 Y. K. Kim, L. S. Gu, T. E. Bryan, J. R. Kim, L. Chen, Y. Liu, J. C. Yoon, L. Breschi, D. H. Pashley and F. R. Tay, Biomaterials, 2010, 31, 6618-6627.

15 Z. Nikfar and Z. Shariatinia, J. Mol. Graphics Modell., 2017, 76, 86-105.

16 Y. Wang, G. Zhu, N. Li, J. Song, L. Wang and X. Shi, Biotechnol. Adv., 2015, 33, 1626-1640.

17 S. E. Kim, Y. P. Yun, K. S. Shim, H. J. Kim, K. Park and H. R. Song, Biomed. Mater., 2016, 11, 55005.

18 N. Cheng, B. T. Estes, T. Young and F. Guilak, Tissue Eng., Part A, 2013, 19, 484-496.

19 X. Zhang, X. Chen, T. Yang, N. Zhang, L. Dong, S. Ma, X. Liu, M. Zhou and B. Li, Cell Tissue Banking, 2014, 15, 531-541.

20 L. P. Yan, Y. J. Wang, L. Ren, G. Wu, S. G. Caridade, J. B. Fan, L. Y. Wang, P. H. Ji, J. M. Oliveira, J. T. Oliveira, J. F. Mano and R. L. Reis, J. Biomed. Mater. Res., Part A, 2010, 95, 465475.
21 J. Kuljanin, I. Jankovic, J. Nedeljkovic, D. Prstojevic and V. Marinkovic, J. Pharm. Biomed. Anal., 2002, 28, 1215-1220.

22 K. Li, J. Wang, X. Liu, X. Xiong and H. Liu, Carbohydr. Polym., 2012, 90, 1573-1581.

23 F. Lu, F. Wang, Z. Chen and H. Huang, Stem Cell Res. Ther., $2017,8,12$.

24 J. Kim, J. Lim, R. Naren, H. Yun and E. K. Park, Acta Biomater., 2016, 44, 155-167.

25 Q. Yao, J. G. Cosme, T. Xu, J. M. Miszuk, P. H. Picciani, H. Fong and H. Sun, Biomaterials, 2017, 115, 115-127.

26 P. Ni, Q. Ding, M. Fan, J. Liao, Z. Qian, J. Luo, X. Li, F. Luo, Z. Yang and Y. Wei, Biomaterials, 2014, 35, 236-248.

27 X. Li and J. Chang, J. Biomed. Mater. Res., Part A, 2008, 85, 293-300.

28 M. Dadsetan, M. Giuliani, F. Wanivenhaus, R. M. Brett, J. E. Charlesworth and M. J. Yaszemski, Acta Biomater., 2012, 8, 1430-1439.

29 I. C. Stancu, R. Filmon, C. Cincu, B. Marculescu, C. Zaharia, Y. Tourmen, M. F. Basle and D. Chappard, Biomaterials, 2004, 25, 205-213.

30 N. R. Gandavarapu, P. D. Mariner, M. P. Schwartz and K. S. Anseth, Acta Biomater., 2013, 9, 4525-4534.

31 T. Takahata, T. Okihara, Y. Yoshida, K. Yoshihara, Y. Shiozaki, A. Yoshida, K. Yamane, N. Watanabe, M. Yoshimura, M. Nakamura, M. Irie, B. Van Meerbeek, M. Tanaka, T. Ozaki and A. Matsukawa, Biomed. Mater., 2015, 10, 65009.

32 H. Mahjoubi, E. Buck, P. Manimunda, R. Farivar, R. Chromik, M. Murshed and M. Cerruti, Acta Biomater., 2017, 47, 149-158.

33 A. Przekora and G. Ginalska, Mater. Sci. Eng. C, 2016, 61, 355-361.

34 S. Kim, Z. Cui, J. Fan, A. Fartash, T. L. Aghaloo and M. Lee, J. Mater. Chem. B, 2016, 4, 5289-5298.

35 Y. H. Wang, E. Rajalakshmanan, C. K. Wang, C. H. Chen, Y. C. Fu, T. L. Tsai, J. K. Chang and M. L. Ho, J. Tissue Eng. Regener. Med., 2017, 11, 2603-2612.

36 Z. Xia, X. Yu, X. Jiang, H. D. Brody, D. W. Rowe and M. Wei, Acta Biomater., 2013, 9, 7308-7319.

37 F. G. Lyons, A. A. Al-Munajjed, S. M. Kieran, M. E. Toner, C. M. Murphy, G. P. Duffy and F. J. O'Brien, Biomaterials, 2010, 31, 9232-9243.

38 T. Sun, S. Yao, M. Liu, Y. Yang, Y. Ji, W. Cui, Y. Qu and X. Guo, Tissue Eng., Part A, 2018, 24, 502-515.

39 Y. Liu, F. Shi, K. Gong, Y. Liu, W. Zhi, J. Weng and S. Qu, J. Mater. Sci.: Mater. Med., 2017, 28, 56.

40 X. Ma, Z. He, F. Han, Z. Zhong, L. Chen and B. Li, Colloids Surf., B, 2016, 143, 81-87.

41 B. M. Watson, T. N. Vo, A. M. Tatara, S. R. Shah, D. W. Scott, P. S. Engel and A. G. Mikos, Biomaterials, 2015, 67, 286-296. 